\title{
FOURIER MULTIPLIERS ON SPACES OF DISTRIBUTIONS
}

\author{
by W. LAMB \\ (Received 3rd October 1984)
}

\section{Introduction}

In [8], Rooney defines a class $\mathscr{A}$ of complex-valued functions $\zeta$ each of which is analytic in a vertical strip $\alpha(\zeta)<\operatorname{Re} s<\beta(\zeta)$ in the complex $s$-plane and satisfies certain growth conditions as $|\operatorname{Im} s| \rightarrow \infty$ along fixed lines $\operatorname{Re} s=c$ lying within this strip. These conditions mean that the functions

$$
\zeta_{\sigma}(t)=\zeta(\sigma+i t), \zeta \in \mathscr{A}, \sigma \in(\alpha(\zeta), \beta(\zeta))
$$

fulfil the requirements of the one-dimensional Mihlin-Hörmander theorem (see $\left[6\right.$, p. 417]) and so can be regarded as Fourier multipliers for the Banach spaces $L^{p}(\mathbb{R})$. Consequently, each function $\zeta \in \mathscr{A}$ gives rise to a family of bounded operators $W[\zeta, \sigma]$, $\sigma \in(\alpha(\zeta), \beta(\zeta))$, on $L^{p}(\mathbb{R}), 1<p<\infty$. By noting that the Mellin transform $M \psi$ of a suitably restricted function $\psi$ could be expressed as

$$
(M \psi)(\mu / p+i t)=\left(F C_{\mu, p} \psi\right)(t)
$$

where $F$ denotes the Fourier transform and

$$
\left(C_{\mu, p} \psi\right)(t)=e^{\mu t / p} \psi\left(e^{t}\right)
$$

(see [7]), Rooney was able to develop a theory of Mellin multipliers on weighted versions of the Banach spaces $L^{p}(0, \infty)$ and as a result deduced the mapping properties of certain operators, including the Erdélyi-Kober operators of fractional integration.

In this paper, functions $\zeta$ in the class $\mathscr{A}$ are shown to generate a family of continuous linear operators $W[\zeta, \sigma], \sigma \in(\alpha(\zeta), \beta(\zeta))$, on the Fréchet spaces $D_{L p}$ introduced by Schwartz in [9]. These operators can be interpreted as the restrictions of the bounded operators on $L^{p}(\mathbb{R})$ mentioned above. An application of standard techniques for extending operators from spaces of classical functions to spaces of generalised functions then establishes that the operators $W[\zeta, \sigma]$ permit natural extensions $\tilde{W}[\zeta, \sigma]$ to the distribution spaces $D_{L^{p}}^{\prime}$. Since each space $D_{L^{p}}^{\prime}(1<p<\infty)$ contains the space $L^{q}(\mathbb{R})$ (where $1 / p+1 / q=1$ ) as a subspace, in the sense of regular generalised functions, the extended operators $\tilde{W}[\zeta, \sigma]$ are also extensions of the corresponding Fourier multipliers on $L^{q}(\mathbb{R})$.

As illustrations of the theory, we concentrate on the Riemann-Liouville and Weyl 
operators of fractional integration and demonstrate how familiar results associated with these operators emerge in a natural manner from properties possessed by their multiplier functions. Also discussed are certain integral operators involving the GaussWeierstrass kernel. The results deduced on all of these operators are used finally to obtain distributional solutions of a class of boundary value problems involving the heat operator.

\section{Notation and preliminary results}

Throughout, $p, q$ and $\mu$ are real numbers with $1<p, q<\infty$ and $1 / p+1 / q=1$. $L^{P}\left(=L^{p}(\mathbb{R})\right)$ denotes the Banach space of (equivalence classes of) functions $\phi$ such that

$$
\int_{-\infty}^{\infty}|\phi(t)|^{p} d t<\infty
$$

with the norm $\|\cdot\|_{p}$ on $L^{p}$ defined in the usual manner by

$$
\|\phi\|_{p}=\left(\int_{-\infty}^{\infty}|\phi(t)|^{p} d t\right)^{1 / p}, \phi \in L^{p} .
$$

For any $\mu$, the space $L_{p, \mu}$ is the weighted version of $L^{p}$ given by

$$
L_{p, \mu}=\left\{\phi: e^{-\mu t} \phi \in L^{p}\right\}
$$

If we introduce a norm $\|\cdot\|_{p, \mu}$ on $L_{p, \mu}$ defined by

$$
\|\phi\|_{p, \mu}=\left\|e^{-\mu t} \phi\right\|_{p}
$$

then $L_{p, \mu}$ becomes a Banach space which is homeomorphic to $L^{p}$ under the homeomorphism $e^{-\mu t}: \phi \rightarrow e^{-\mu t} \phi, \phi \in L_{p, \mu}$.

We shall also be concerned with certain subspaces $D_{L^{p}}$ and $D_{p, \mu}$ of $L^{p}$ and $L_{p, \mu}$ respectively. These are defined by

$$
D_{L^{p}}=\left\{\phi \in C^{\infty}(\mathbb{R}): D^{k} \phi \in L^{p}, k=0,1, \ldots\right\}, \quad D_{p, \mu}=\left\{\phi \in C^{\infty}(\mathbb{R}): e^{-\mu t} \phi \in D_{L p}\right\} .
$$

Equipped with the topologies generated by the families of seminorms $\left\{v_{k}^{p}\right\}$ and $\left\{v_{k}^{p, \mu}\right\}$ $(k=0,1, \ldots)$ respectively, where

$$
v_{k}^{p}(\phi)=\left\|D^{k} \phi\right\|_{p}, \phi \in D_{L \rho}, \quad v_{k}^{p, \mu}(\phi)=\left\|D^{k}\left(e^{-\mu t} \phi\right)\right\|_{p}=v_{k}^{p}\left(e^{-\mu t} \phi\right), \phi \in D_{p, \mu},
$$

$D_{L p}$ and $D_{p, \mu}$ are Fréchet spaces, with $D_{L p}$ and $D_{p, 0}$ identical. In addition, for each pair of parameters $(p, \mu), D_{p, \mu}$ is a space of test functions in the sense of Zemanian [11] and consequently functionals in the dual $D_{p, \mu}^{\prime}$ can be regarded as generalised functions. Convergence in $D_{p, \mu}^{\prime}$ is defined by means of the weak*-topology. Hence, if $(f, \phi)$ denotes the action of $f \in D_{p, \mu}^{\prime}$ on $\phi \in D_{p, \mu}$ then $\left\{f_{n}\right\} \subset D_{p, \mu}^{\prime}$ converges to $f \in D_{p, \mu}$ if and only if $\left(f_{n}, \phi\right) \rightarrow(f, \phi)$ in $\mathbb{C}$ for each $\phi \in D_{p, \mu}$. 
Properties of the spaces $D_{p, \mu}$ and $D_{p, \mu}^{\prime}$ can easily be obtained from the corresponding properties of $D_{L^{p}}$ and $D_{L^{p}}^{\prime}$ given in [9] (see [3]). We list the main facts below.

Lemma 2.1. If $\phi \in D_{p, \mu}$ then $|\phi(t)| \leqq M e^{\mu t}$ for all $t \in \mathbb{R}$, where $M$ is a positive constant independent of $t$.

Lemma 2.2. The mapping $e^{\lambda t}$ defined by $e^{\lambda t}: \phi \rightarrow e^{\lambda t} \phi$ is a homeomorphism of $D_{p, \mu}$ onto $D_{p, \mu+\lambda}$ with inverse $e^{-\lambda t}$ for any real numbers $\lambda$ and $\mu$.

Lemma 2.3. The mapping $e^{\widetilde{\lambda t}}$ defined on $D_{p, \mu}^{\prime}$ for any $\mu, \lambda \in \mathbb{R}$ by

$$
\left(\widetilde{e^{\lambda t}} f, \phi\right)=\left(f, e^{\lambda t} \phi\right) f \in D_{p, \mu}^{\prime}, \phi \in D_{p, \mu-\lambda}
$$

is a homeomorphism of $D_{p, \mu}^{\prime}$ onto $D_{p, \mu-\lambda}^{\prime}$ with inverse $\widetilde{e^{-\lambda t}}$.

Lemma 2.4. If $\psi \in L_{q,-\mu}$ then $\psi$ generates a regular generalised function $\tilde{\psi} \in D_{p, \mu}^{\prime}$ by means of the formula

$$
(\tau, \phi)=\int_{-\infty}^{\infty} \psi(x) \phi(x) d x, \phi \in D_{p, \mu}
$$

Lemma 2.5. If $f \in D_{p, \mu}^{\prime}$ then $f=\sum_{k=0}^{n} \tilde{D}^{k} \mathcal{\psi}_{k}$ where, for $k=0,1, \ldots, n, \psi_{k} \in L_{q,-\mu}$ and $\tilde{D}^{k}$ denotes the distributional differential operator defined by

$$
\left(\tilde{D}^{k} f, \phi\right)=(-1)^{k}\left(f, D^{k} \phi\right), f \in D_{p, \mu}^{\prime}, \phi \in D_{p, \mu} .
$$

Note that (2.3) is meaningful since $D^{k}$ can be shown to be a continuous linear mapping of $D_{p, \mu}$ into $D_{p, \mu}$ for any $\mu$.

\section{Fourier multipliers}

Definition 3.1. A measurable function $\Psi: \mathbb{R} \rightarrow \mathbb{C}$ is a Fourier multiplier for $L^{p}$ (henceforth abbreviated to an $L^{p}$ multiplier) if there exists a bounded operator $W[\Psi]: L^{p} \rightarrow L^{p}$ such that

$$
F(W[\Psi] \phi)=\Psi[F \phi], \phi \in L^{2} \cap L^{p}
$$

Here, and in the sequel, $F$ denotes the Fourier transform defined on $L^{1}$ by

$$
(F \phi)(t)=\int_{-\infty}^{\infty} e^{i i x} \phi(x) d x
$$

and extended by continuity from $L^{1} \cap L^{2}$ to $L^{2}$ (see [6, p. 305]).

Of particular interest is the following class of multipliers introduced by Rooney [8]. 
Definition 3.2. The function $\zeta: \mathbb{C} \rightarrow \mathbb{C}$ belongs to the class $\mathscr{A}$ if there exist extended real numbers $\alpha(\zeta)$ and $\beta(\zeta)$ such that

(a) $\zeta$ is analytic in the strip $\alpha(\zeta)<\operatorname{Re} s<\beta(\zeta)$,

(b) $\zeta$ is bounded in every closed substrip $\sigma_{1} \leqq \operatorname{Re} s \leqq \sigma_{2}$, where $\alpha(\zeta)<\sigma_{1} \leqq \sigma_{2}<\beta(\zeta)$,

(c) $\left|\zeta^{\prime}(\sigma+i t)\right|=O\left(|t|^{-1}\right)$ as $|t| \rightarrow \infty$ for $\alpha(\zeta)<\sigma<\beta(\zeta)$.

Theorem 3.3. Let $\zeta$ be a function in $\mathscr{A}$ and let $\zeta_{\sigma}$ be defined by

$$
\zeta_{\sigma}(t)=\zeta(\sigma+i t), t \in \mathbb{R}
$$

Then, for each $\sigma \in(\alpha(\zeta), \beta(\zeta))$ and $p \in(1, \infty), \zeta_{\sigma}$ is an $L^{p}$ multiplier i.e. for each $\sigma \in(\alpha(\zeta), \beta(\zeta))$ and $p \in(1, \infty)$ there exists a bounded operator $W[\zeta, \sigma]: L^{p} \rightarrow L^{p}$ such that

$$
F(W[\zeta, \sigma] \phi)=\zeta_{\sigma}[F \phi], \phi \in L^{2} \cap L^{p} .
$$

Proof. See [8].

Theorem 3.4. If $\zeta$ belongs to the class $\mathscr{A}$ then, for each $\sigma \in(\alpha(\zeta), \beta(\zeta))$ and $p \in(1, \infty)$, the function $\zeta_{\sigma}^{*}$ defined by

$$
\zeta_{a}^{*}(t)=\zeta(\sigma-i t)=\zeta^{*}(\sigma+i t)
$$

(where $\zeta^{*}(s)=\zeta(\vec{s})$ ) is an $L^{p}$ multiplier i.e. for each $\sigma \in(\alpha(\zeta), \beta(\zeta))$ and $p \in(1, \infty)$, there exists a bounded operator $W\left[\zeta^{*}, \sigma\right]: L^{p} \rightarrow L^{p}$ such that

$$
F\left(W\left[\zeta^{*}, \sigma\right] \phi\right)=\zeta_{\sigma}^{*}[F \phi], \phi \in L^{2} \cap L^{p}
$$

Proof. Since $\zeta \in \mathscr{A}$ it follows that, for each $\sigma \in(\alpha(\zeta), \beta(\zeta)), \quad \zeta_{\sigma}^{*} \in L^{\infty}(\mathbb{R}) \cap C^{1}(\mathbb{R})$. Moreover, from Definition 3.2(c), $\left|\left(D \zeta_{\sigma}^{*}\right)(t)\right|=O\left(|t|^{-1}\right)$ as $|t| \rightarrow \infty$ and therefore there exists a positive constant $c$ such that, for each $r>0$,

$$
\int_{I_{r}}\left|\zeta_{\sigma}^{*}(t)\right|^{2} d t \leqq c^{2} r
$$

and

$$
\int_{I_{r}}\left|\left(D \zeta_{\sigma}^{*}\right)(t)\right|^{2} d t \leqq c^{2} r^{-1}
$$

where $I_{r}=\{t: r / 2<|t|<r\}$. The result now follows immediately from the MihlinHörmander theorem (see [6, p. 417]).

Our aim in this section is to establish that the restrictions of the operators $W[\zeta, \sigma]$ and $W\left[\zeta^{*}, \sigma\right]$ to the space $D_{L^{p}}$ are continuous linear mappings of $D_{L^{p}}$ into $D_{L^{p}}$ whenever $\zeta \in \mathscr{A}, \sigma \in(\alpha(\zeta), \beta(\zeta))$ and $p \in(1, \infty)$. To this end, we give the following preliminary results and definitions.

Definition 3.5. The set $\Theta_{M}$ of functions of slow growth consists of functions $\theta \in C^{\infty}(\mathbb{R})$ such that, for each $k=0,1, \ldots$, there exists a non-negative integer $N_{k}$ with

$$
\sup _{t \in \mathbb{R}}\left(1+t^{2}\right)^{-N_{k}}\left|\left(D^{k} \phi\right)(t)\right|<\infty
$$


Lemma 3.6. If $\theta \in \Theta_{M}$ and $\psi \in S$, the space of test functions of rapid descent, then $\theta \psi \in S$.

Proof. See $[10$, p. 107].

Lemma 3.7. If $\zeta \in \mathscr{A}$ then $\zeta_{\sigma}$ and $\zeta_{\sigma}^{*}$ both belong to the class $\Theta_{M}$ for each $\sigma \in(\alpha(\zeta), \beta(\zeta))$.

Proof. Let $\sigma \in(\alpha(\zeta), \beta(\zeta))$ be arbitrarily fixed and consider the function $\zeta_{\sigma}$ defined by (3.1). Since $\zeta$ is analytic in the strip $\alpha(\zeta)<\operatorname{Re} s<\beta(\zeta), \zeta_{\sigma}$ clearly belongs to the class $C^{\infty}(\mathbb{R})$. Moreover, if $s^{\prime}$ is any point on the line $\operatorname{Re} s=\sigma$ then there exists an $\varepsilon>0$ such that the circle $\left\{s:\left|s-s^{\prime}\right|<\varepsilon\right\} \subset\{s: \alpha(\zeta)<\operatorname{Re} s<\beta(\zeta)\}$ and from Cauchy's inequality

$$
\left|\zeta^{(k)}\left(s^{\prime}\right)\right| \leqq M\left(\varepsilon, s^{\prime}, \sigma\right) k ! \varepsilon^{-k} \quad(k=0,1, \ldots)
$$

where $M\left(\varepsilon, s^{\prime}, \sigma\right)$ denotes the maximum value of $\zeta$ on the circle. From Definition 3.2(b), $\zeta$ is uniformly bounded on the strip $\sigma-\varepsilon \leqq \operatorname{Re} s \leqq \sigma+\varepsilon$ and therefore the constant $M\left(\varepsilon, s^{\prime}, \sigma\right)$ in (3.3) is independent of the choice of $s^{\prime}$ on the line $\operatorname{Re} s=\sigma$. Hence $\zeta_{\sigma} \in \Theta_{M}$. Similarly, since $\left|\left(D^{k} \zeta_{\sigma}^{*}\right)(t)\right|=\left|\left(D^{k} \zeta_{\sigma}\right)(-t)\right|$ for $k=0,1, \ldots$, we can conclude that $\zeta_{\sigma}^{*}$ is also a function in $\Theta_{M}$ for each $\sigma \in(\alpha(\zeta), \beta(\zeta))$.

Theorem 3.8. Let $\zeta \in \mathscr{A}$ and, for each $\sigma \in(\alpha(\zeta), \beta(\zeta))$, let $W[\zeta, \sigma]$ and $W\left[\zeta^{*}, \sigma\right]$ be the bounded operators on $L^{p}$ corresponding to the multipliers $\zeta_{\sigma}$ and $\zeta_{\sigma}^{*}$ respectively. Then, for each $p \in(1, \infty)$, the restrictions of $W[\zeta, \sigma]$ and $W\left[\zeta^{*}, \sigma\right]$ to $D_{L^{p}}$ are continuous linear mappings of $D_{L^{p}}$ into $D_{L^{p}}$.

Proof. Let $\sigma \in(\alpha(\zeta), \beta(\zeta))$ be fixed and let $\psi \in S$ so that, by Theorem 3.3,

$$
F(W[\zeta, \sigma] \psi)=\zeta_{\sigma}[F \psi]
$$

From Lemmas 3.6 and 3.7 and the fact that $F: S \rightarrow S$ is a homeomorphism, we can deduce that the right-hand side of (3.4) is a function in $S$ and therefore, by choosing $F^{-1} \zeta_{\sigma} F \psi(\in S)$ as the representative of the equivalence class $W[\zeta, \sigma] \psi$ in $L^{p}$, we can write $W[\zeta, \sigma] \psi=F^{-1} \zeta_{\sigma} F \psi$. Consequently, for $k=0,1, \ldots$

$$
\begin{aligned}
\left(D^{k} W[\zeta, \sigma] \psi\right)(t) & =(2 \pi)^{-1} D^{k} \int_{-\infty}^{\infty} e^{-i x t} \zeta_{\sigma}(x) \int_{-\infty}^{\infty} e^{i y x} \psi(y) d y d x \quad(t \in \mathbb{R}) \\
& =(2 \pi)^{-1} \int_{-\infty}^{\infty} e^{-i x t} \zeta_{\sigma}(x)(-i x)^{k} \int_{-\infty}^{\infty} e^{i y x} \psi(y) d y d x \quad \text { (by [10, p. 182(3)]) } \\
& =(2 \pi)^{-1} \int_{-\infty}^{\infty} e^{-i x t} \zeta_{\sigma}(x) \int_{-\infty}^{\infty} e^{i y x}\left(D^{k} \psi\right)(y) d y d x \quad \text { (by [10, p. 182(4)]) } \\
& =\left(W[\zeta, \sigma] D^{k} \psi\right)(t) .
\end{aligned}
$$


Thus $D^{k} W[\zeta, \sigma] \psi=W[\zeta, \sigma] D^{k} \psi$ for any $\psi \in S$. Now let $\phi$ be any function in $D_{L p}$. Since $S$ is dense in $D_{L p}$, there exists a sequence $\left\{\psi_{n}\right\}$ of functions in $S$ such that $\psi_{n} \rightarrow \phi$ in $D_{L^{p}}$. By the result proved above,

$$
\begin{aligned}
v_{k}^{p}\left(W[\zeta, \sigma]\left(\psi_{n}-\psi_{m}\right)\right) & =\left\|D^{k} W[\zeta, \sigma]\left(\psi_{n}-\psi_{m}\right)\right\|_{p} \\
& =\left\|W[\zeta, \sigma] D^{k}\left(\psi_{n}-\psi_{m}\right)\right\|_{p} \\
& \leqq\|W[\zeta, \sigma]\| v_{k}^{p}\left(\psi_{n}-\psi_{m}\right) \rightarrow 0 \quad \text { as } n, m \rightarrow \infty,
\end{aligned}
$$

and therefore $\left\{W[\zeta, \sigma] \psi_{n}\right\}$ is a Cauchy sequence in $D_{L^{p}}$. Since $D_{L^{p}}$ is complete, this means that a function $\xi \in D_{L^{p}}$ exists such that $W[\zeta, \sigma] \psi_{n} \rightarrow \xi$ in $D_{L^{p}}$. If we extend $W[\zeta, \sigma]$ from $S$ to $D_{L^{p}}$ by setting $\xi=W[\zeta, \sigma] \phi$ then it follows that

$$
D^{k} W[\zeta, \sigma] \phi=W[\zeta, \sigma] D^{k} \phi
$$

for each $\phi \in D_{L^{p}}$ and $k=0,1,2, \ldots$. Therefore, the operator $W[\zeta, \sigma]$, thus extended, is a continuous linear mapping of $D_{L^{p}}$ into $D_{L^{p}}$. Finally, since convergence in $D_{L^{p}}$ implies convergence in $L^{p}$, we can state that, for $\phi \in D_{L^{p}}, W[\zeta, \sigma] \phi$, as defined above, agrees almost everywhere on $(-\infty, \infty)$ with $W[\zeta, \sigma] \phi$ where, in the latter, $W[\zeta, \sigma]$ is interpreted as the bounded operator on $L^{p}$ corresponding to the multiplier $\zeta_{\sigma}$. This completes the proof for $W[\zeta, \sigma]$ and the proof for $W\left[\zeta^{*}, \sigma\right]$ follows similarly.

\section{Fractional integrals on $D_{p, \mu}$}

In this section we shall determine the mapping properties of the Riemann-Liouville and Weyl operators of fractional calculus on the spaces $D_{p, \mu}$. For $\operatorname{Re} a>0$, the RiemannLiouville and Weyl fractional integrals of order $a$ are defined on suitable functions $\phi$ by

$$
\begin{array}{ll}
\left(I^{a} \phi\right)(t)=(\Gamma(a))^{-1} \int_{-\infty}^{t}(t-\tau)^{a-1} \phi(\tau) d \tau, & -\infty<t<\infty, \\
\left(K^{a} \phi\right)(t)=(\Gamma(a))^{-1} \int_{t}^{\infty}(\tau-t)^{a-1} \phi(\tau) d \tau, & -\infty<t<\infty,
\end{array}
$$

respectively, while the operators of fractional differentiation $D^{a}$ and $E^{a}$ are given by

$$
\begin{gathered}
\left(D^{a} \phi\right)(t)=\left(I^{n-a} D^{n} \phi\right)(t), \quad-\infty<t<\infty, \\
\left(E^{a} \phi\right)(t)=(-1)^{n}\left(K^{n-a} D^{n} \phi\right)(t), \quad-\infty<t<\infty,
\end{gathered}
$$

where $n$, a positive integer, is such that $n>\operatorname{Re} a$. Results concerning these operators on the spaces $D_{p, \mu}$ have been deduced previously by the author [3] as a special case of a theory of fractional powers of operators. Here we shall show that the same results emerge in a natural manner from properties possessed by associated multiplier functions. 
Of interest also are generalisations $I_{b}^{a}$ and $K_{b}^{a}$ of $I^{a}$ and $K^{a}$ respectively. For $a \in \mathbb{C}$ and $b>0, I_{b}^{a}$ and $K_{b}^{a}$ are defined on suitable functions $\phi$ by

$$
\begin{array}{ll}
\left(I_{b}^{a} \phi\right)(t)=(\Gamma(a))^{-1} \int_{-\infty}^{t}(t-\tau)^{a-1} \exp (-b / 4(t-\tau)) \phi(\tau) d \tau, & -\infty<t<\infty \\
\left(K_{b}^{a} \phi\right)(t)=(\Gamma(a))^{-1} \int_{i}^{\infty}(\tau-t)^{a-1} \exp (-b / 4(\tau-t)) \phi(\tau) d \tau, & -\infty<t<\infty
\end{array}
$$

As with the operators of fractional calculus defined above, properties of $I_{b}^{a}$ and $K_{b}^{a}$ on $D_{p, \mu}$ will be deduced from the results proved on multipliers in the previous section.

We begin by establishing the mapping properties of $I^{a}, K^{a}, I_{b}^{a}$ and $K_{b}^{a}$ on the Banach spaces $L_{p, \mu}$.

Theorem 4.1. Let $\mu>0$ and $p \in(1, \infty)$.

(a) If $\operatorname{Re} a>0$ then $I^{a}$ is a bounded linear operator mapping $L_{p, \mu}$ into $L_{p, \mu}$.

(b) If $a \in \mathbb{C}$ and $b>0$ then $I_{b}^{a}$ is a bounded linear operator mapping $L_{p, \mu}$ into $L_{p, \mu}$.

(c) $I_{b}^{1 / 2}$ converges to $I^{1 / 2}$ in the operator norm topology as $b \rightarrow 0+$.

\section{Proof.}

(a) For $\mu>0, \operatorname{Re} a>0$ and $\phi \in L_{p, \mu}$,

$$
\begin{aligned}
\left\|I^{a} \phi\right\|_{p, \mu} & =\left\|e^{-\mu t} I^{a} e^{\mu t} \psi\right\|_{p} \quad\left(\psi \in L^{p}, \psi=e^{-\mu t} \phi\right) \\
& =\left\|\left(H(t) e^{-\mu t} t^{a-1} / \Gamma(a)\right) * \psi\right\|_{p} \quad(H \text { the Heaviside function }) \\
& \leqq G_{a}(\mu)\|\psi\|_{p}=G_{a}(\mu)\|\phi\|_{p, \mu} \quad \text { (by Young's inequality) }
\end{aligned}
$$

where $G_{a}(\mu)$, the $L^{1}$ norm of the function $H(t) e^{-\mu t} t^{a-1} / \Gamma(a)$ is given by

$$
G_{a}(\mu)=\Gamma(\operatorname{Re} a) /\left(|\Gamma(a)| \mu^{\operatorname{Rea}}\right) \quad[2, \text { p. } 137(1)]
$$

(b) Proceeding as in the proof of (a), this time with $a \in \mathbb{C}$, we can show that

$$
\left\|I_{b}^{a} \phi\right\|_{p, \mu} \leqq G_{a, b}(\mu)\|\phi\|_{p, \mu} \quad(b>0)
$$

where

$$
G_{a, b}(\mu)=2(b / 4 \mu)^{\operatorname{Re} a / 2}|\Gamma(a)|^{-1} K_{\operatorname{Re} a}(\sqrt{b \mu}) \quad(b>0) \quad[2, \text { p. 146(29)] }
$$

The function $K_{v}(z)$ appearing in the above formula is the modified Bessel function of the third kind (see [4, p. 108]). 
(c) Since $K_{1 / 2}(z)=(\pi / 2 z)^{1 / 2} e^{-z}[4$, p. 112] we can apply Young's inequality to deduce that

$$
\begin{aligned}
& \left\|I^{1 / 2} \phi-I_{b}^{1 / 2} \phi\right\|_{p, \mu} \quad\left(\phi \in L_{p, \mu}\right) \\
& \leqq \mu^{-1 / 2}(1-\exp (-\sqrt{b \mu}))\|\phi\|_{p, \mu} \rightarrow 0 \text { as } b \rightarrow 0+,
\end{aligned}
$$

as required.

Theorem 4.2. Let $\mu<0$ and $p \in(1, \infty)$.

(a) If $\operatorname{Re} a>0$ then $K^{a}$ is a bounded linear operator mapping $L_{p, \mu}$ into $L_{p, \mu}$.

(b) If $a \in \mathbb{C}$ and $b>0$ then $K_{b}^{a}$ is a bounded linear operator mapping $L_{p, \mu}$ into $L_{p, \mu}$.

(c) $K_{b}^{1 / 2}$ converges to $K^{1 / 2}$ in the operator norm topology as $b \rightarrow 0+$.

Proof. The proof of this theorem is similar to that of Theorem 4.1.

\section{Theorem 4.3.}

(a) If $\mu>0, p \in(1, \infty)$ and $\operatorname{Re} a>0$ then $I^{a}$ is a homeomorphism of $D_{p, \mu}$ onto $D_{p, \mu}$ with $\left(I^{a}\right)^{-1}=D^{a}$.

(b) If $\mu<0, p \in(1, \infty)$ and $\operatorname{Re} a>0$ then $K^{a}$ is a homeomorphism of $D_{p, \mu}$ onto $D_{p, \mu}$ with $\left(K^{a}\right)^{-1}=E^{a}$.

\section{Proof.}

(a) In view of Lemma 2.2 and the definition of the topology on $D_{p, \mu}$, it is sufficient to prove that, for $\mu>0, e^{-\mu t} I^{a} e^{\mu t}$ is a homeomorphism of $D_{L^{p}}$ onto $D_{L^{p}}$ with inverse given by $e^{-\mu t} D^{a} e^{\mu t}$. To this end, let $\zeta$ be the function defined by

$$
\zeta(s)=s^{-a}=\exp (-a \log s)
$$

where the function $\log s$ assumes its principal branch and $s=\sigma+i t$. Since $\operatorname{Re} a>0, \zeta$ is clearly analytic in the half-plane $\operatorname{Re} s>0$ and is bounded on every substrip of the form $\sigma_{1} \leqq \operatorname{Re} s \leqq \sigma_{2}$ where $0<\sigma_{1} \leqq \sigma_{2}<\infty$. In addition, $\zeta^{\prime}(s)=-a s^{-a} / s$ so that

$$
\left|\zeta^{\prime}(\sigma+i t)\right|=\left|a(\sigma+i t)^{-a}\right| /\left(\sigma^{2}+t^{2}\right)^{1 / 2}=O\left(|t|^{-1}\right) \text { as }|t| \rightarrow \infty
$$

for each $\sigma \in(0, \infty)$. It follows from this that $\zeta \in \mathscr{A}$ with $\alpha(\zeta)=0, \beta(\zeta)=\infty$ and therefore, by Theorem 3.4, there exists, for each $\sigma \in(0, \infty)$ and $p \in(1, \infty)$, a continuous linear operator $W\left[\zeta^{*}, \sigma\right]: D_{L P} \rightarrow D_{L p}$ such that

$$
\begin{aligned}
\left(F W\left[\zeta^{*}, \sigma\right] \psi\right)(t) & =\zeta_{\sigma}^{*}(t)(F \psi)(t) \\
& =(\sigma-i t)^{-a}(F \psi)(t), \psi \in S
\end{aligned}
$$


Since

$$
\begin{aligned}
{\left[F\left(e^{-\mu x} I^{a} e^{\mu x} \psi\right)\right](t) } & =\left[F\left(e^{-\mu x} x^{a-1} H(x) / \Gamma(a)\right)\right](t)(F \psi)(t) \\
& =(\mu-i t)^{-a}(F \psi)(t), \psi \in S
\end{aligned}
$$

we can deduce that, for each $\mu \in(0, \infty)$,

$$
W\left[\zeta^{*}, \mu\right]=e^{-\mu t} I^{a} e^{\mu t}
$$

as operators on $D_{L^{p}}$ and hence $I^{a}$ is a continuous linear mapping of $D_{p, \mu}$ into $D_{p, \mu}$ for any $\mu>0$. Finally, to demonstrate that $I^{a}$ is a homeomorphism of $D_{p, \mu}$ onto $D_{p, \mu}$ with inverse $D^{a}$ we examine the function $\eta(s)=1 / \zeta^{*}(s)=(\sigma-i t)^{a}$. This function is clearly not in the class $\mathscr{A}$. However, if we note that $s^{a}$ can be decomposed into the product $s^{-n+a} s^{n}$ where $n$, a positive integer, is such that $n>\operatorname{Re} a$, then, since

$$
\left[F\left(e^{-\mu x} D^{n} e^{\mu x} \psi\right)\right](t)=(\mu-i t)^{n}(F \psi)(t), \psi \in S, n=1,2, \ldots,
$$

and $e^{-\mu t} D^{n} e^{\mu t}$, for each $n=1,2, \ldots$, is a continuous linear mapping of $D_{L^{p}}$ into $D_{L^{p}}$, it follows that, for each $\mu>0$ and $p \in(1, \infty)$, there exists a continuous linear operator $W[\eta, \mu]: D_{L^{p}} \rightarrow D_{L^{p}}$ with the property that

$$
(F W[\eta, \mu] \psi)(t)=(\mu-i t)^{a}(F \psi)(t), \psi \in S
$$

By the results proved above and the definition of $D^{a}$,

$$
W[\eta, \mu]=e^{-\mu t} I^{n-a} D^{n} e^{\mu t}=e^{-\mu t} D^{a} e^{\mu t}
$$

and consequently, $D^{a}: D_{p, \mu} \rightarrow D_{p, \mu}$ is a continuous linear mapping if $\mu>0$. Moreover, since

$$
\begin{aligned}
W[\eta, \mu] W\left[\zeta^{*}, \mu\right] \psi(t) & =\left(F^{-1}(\mu-i t)^{a}(\mu-i t)^{-a} F \psi\right)(t) \\
& =\psi(t), \psi \in S,
\end{aligned}
$$

it follows that $W[\eta, \mu]=\left(W\left[\zeta^{*}, \mu\right]\right)^{-1}$ on $D_{L^{p}}$ and this completes the proof.

(b) This can be proved similarly by verifying that, for $\mu<0, e^{-\mu t} K^{a} e^{\mu t}=W[\zeta,-\mu]$ as operators on $D_{L p \text {, }}$ where $\zeta(s)=s^{-a}$.

\section{Theorem 4.4.}

(a) If $\mu>0, p \in(1, \infty)$ and $b>0$ then $I_{b}^{a}$ is a continuous linear mapping of $D_{p, \mu}$ into $D_{p, \mu}$ for any $a \in \mathbb{C}$.

(b) If $\mu<0, p \in(1, \infty)$ and $b>0$ then $K_{b}^{a}$ is a continuous linear mapping of $D_{p, \mu}$ into $D_{p, \mu}$ for any $a \in \mathbb{C}$. 
Proof. As in the proof of Theorem 4.3, it is sufficient to verify that $e^{-\mu t} I_{b}^{a} e^{\mu t}$ is a continuous linear mapping of $D_{L^{p}}$ into $D_{L^{p}}$ for any $p \in(1, \infty), \mu>0, b>0$ and $a \in \mathbb{C}$. Clearly, from Theorem 4.1(b), $e^{-\mu t} I_{b}^{a} e^{\mu t}$ is a bounded operator mapping $L^{p}$ into $L^{p}$. Moreover, for $\phi \in D_{L p}$,

$$
\begin{aligned}
\left|e^{-\mu t} I_{b}^{a} e^{\mu t} \phi(t)\right| & \leqq|\Gamma(a)|^{-1} \int_{-\infty}^{t}(t-\tau)^{\operatorname{Re} a-1} e^{-\mu(t-\tau)} e^{-b / 4(t-\tau)}|\phi(\tau)| d \tau \\
& \leqq M|\Gamma(a)|^{-1} \int_{0}^{\infty} e^{-\mu u} u^{\operatorname{Re} a-1} e^{-b / 4 u} d u \quad \text { (by Lemma 2.1) } \\
& =2 M|\Gamma(a)|^{-1}(b / 4 \mu)^{\operatorname{Re} a / 2} K_{\operatorname{Re} a}\left(b^{1 / 2} \mu^{1 / 2}\right) \quad(\text { by }[2, \text { p. 146(29)]) }
\end{aligned}
$$

and so $e^{-\mu t} I_{b}^{a} e^{\mu t} \phi$ is continuous on $\mathbb{R}$ for each $\phi \in D_{L^{p}}$. Now consider the function $\zeta(s)=2[\Gamma(a)]^{-1}(b / 4 s)^{a / 2} K_{a}\left(b^{1 / 2} s^{1 / 2}\right)$. This function is analytic in the half-plane $\operatorname{Re} s>0$. Moreover, as $s \rightarrow \infty$,

$$
(2 s / \pi)^{1 / 2} e^{s} K_{v}(s)=0\left(|s|^{-1}\right) \quad(|\arg s|<\pi-\delta, \delta>0)
$$

(from [4, p. 123, (5.11.9)]) and therefore $\zeta(s)$ is bounded in every closed substrip $\sigma_{1} \leqq \operatorname{Re} s \leqq \sigma_{2}$ where $0<\sigma_{1} \leqq \sigma_{2}<\infty$. Similarly, since

$$
\frac{d}{d s}\left(s^{v} K_{v}(s)\right)=-s^{v} K_{v-1}(s)
$$

we can deduce that $\left|\zeta^{\prime}(\sigma+i t)\right|=O\left(|t|^{-1}\right)$ as $|t| \rightarrow \infty$ for $0<\sigma<\infty$. Consequently $\zeta \in \mathscr{A}$ with $\alpha(\zeta)=0, \beta(\zeta)=\infty$ and so, for each $\sigma>0$ and $p \in(1, \infty)$, there exist continuous linear operators $W[\zeta, \sigma]$ and $W\left[\zeta^{*}, \sigma\right]$ mapping $D_{L^{p}}$ into $D_{L^{p}}$ such that

$$
F(W[\zeta, \sigma] \phi)=\zeta_{\sigma}[F \phi]
$$

and

$$
F\left(W\left[\zeta^{*}, \sigma\right] \phi\right)=\zeta_{\sigma}^{*}[F \phi](\phi \in S)
$$

If we let $\phi \in S$ and examine $F\left(e^{-\mu t} I_{b}^{a} e^{\mu t} \phi\right)$ then we find that

$$
\begin{aligned}
F\left(e^{-\mu x} I_{b}^{a} e^{\mu x} \phi\right)(t) & =(\Gamma(a))^{-1}\left[F\left(e^{-\mu x} e^{-b / 4 x} x^{a-1} H(x)\right)\right](t)(F \phi)(t) \\
& =2(\Gamma(a))^{-1}(b / 4(\mu-i t))^{a / 2} K_{a}\left(b^{1 / 2}(\mu-i t)^{1 / 2}\right)(F \phi)(t) \quad[4, \text { p. 146, (29) }] \\
& =\zeta_{\mu}^{*}(t)(F \phi)(t) .
\end{aligned}
$$

This implies that $W\left[\zeta^{*}, \mu\right]=e^{-\mu t} I_{b}^{a} e^{\mu t}$ as bounded operators on $L^{p}$ and therefore, for $\phi \in D_{L P}$,

$$
W\left[\zeta^{*}, \mu\right] \phi=e^{-\mu t} I_{b}^{a} e^{\mu t} \phi
$$


a.e. on $\mathbb{R}$. In fact, since each side of (4.6) is a continuous function on $\mathbb{R}$, we can state that $W\left[\zeta^{*}, \mu\right] \phi \equiv e^{-\mu t} I_{b}^{a} e^{\mu t} \phi$ on $\mathbb{R}$ and this proves (a). Similarly, if we examine $F\left[e^{-\mu x} K_{b}^{a} e^{\mu x} \phi\right]$ for $\phi \in S$ then

$$
\left[F\left(e^{-\mu x} K_{b}^{a} e^{\mu x} \phi\right)\right](t)=\zeta_{-\mu}(t)(F \phi)(t), \mu<0,
$$

and arguing as above, we conclude that $K_{b}^{a}: D_{p, \mu} \rightarrow D_{p, \mu}$ is continuous for $\mu<0$.

For future reference, we list the results obtained in Theorems $4.1-4.4$ in the following table.

Table 4.5

\begin{tabular}{|c|c|c|c|c|}
\hline Operator & $\begin{array}{l}\text { Corresponding } \\
\text { multiplier operator }\end{array}$ & Multiplier & Properties & $\begin{array}{c}\text { Restrictions on } \\
\text { parameters }\end{array}$ \\
\hline$I^{a}$ & $e^{\mu t} W\left[\zeta^{*}, \mu\right] e^{-\mu t}$ & $\stackrel{s^{-a}}{\operatorname{Re} s>0}$ & $\begin{array}{c}\text { Bounded on } \\
L_{p, \mu \cdot} \\
\text { Homeomorphism } \\
\text { of } D_{p, \mu} \text { onto } \\
D_{p, \mu} \text { with } \\
\left(I^{a}\right)^{-1}=D^{a} .\end{array}$ & $\begin{array}{c}\operatorname{Re} a>0 \\
\mu>0 \\
p \in(1, \infty)\end{array}$ \\
\hline$K^{a}$ & $e^{\mu t} W[\zeta,-\mu] e^{-\mu t}$ & $\begin{array}{c}s^{-a} \\
\operatorname{Re} s>0\end{array}$ & $\begin{array}{l}\text { Bounded on } L_{p, \mu} \\
\text { Homeomorphism } \\
\text { of } D_{p, \mu} \text { onto } D_{p, \mu} \\
\text { with }\left(K^{a}\right)^{-1}=E^{a} .\end{array}$ & $\begin{array}{c}\operatorname{Re} a>0 \\
\mu<0 \\
p \in(1, \infty)\end{array}$ \\
\hline$D^{\sigma}$ & $e^{\mu t} W\left[\zeta^{*}, \mu\right] e^{-\mu t}$ & $\begin{array}{c}s \\
\operatorname{Re} s>0\end{array}$ & $\begin{array}{l}\text { Homeomorphism } \\
\text { of } D_{p, \mu} \text { onto } D_{p, \mu} \\
\text { with }\left(D^{a}\right)^{-1}=I^{a} \text {. }\end{array}$ & $\begin{array}{c}\operatorname{Re} a>0 \\
\mu>0 \\
p \in(1, \infty)\end{array}$ \\
\hline$E^{a}$ & $e^{\mu t} W[\zeta,-\mu] e^{-\mu t}$ & $\begin{array}{r}s, \\
\operatorname{Re} s>0\end{array}$ & $\begin{array}{l}\text { Homeomorphism } \\
\text { of } D_{p, \mu} \text { onto } D_{p, \mu} \\
\text { with }\left(E^{a}\right)^{-1}=K^{a} .\end{array}$ & $\begin{array}{c}\operatorname{Re} a>0 \\
\mu<0 \\
p \in(1, \infty)\end{array}$ \\
\hline$I_{b}^{a}$ & $e^{\mu t} W\left[\zeta^{*}, \mu\right] e^{-\mu t}$ & $\begin{array}{c}\frac{2(b / 4 s)^{a / 2} K_{a}\left(b^{1 / 2} s^{1 / 2}\right)}{\Gamma(a)} \\
\operatorname{Re} s>0\end{array}$ & $\begin{array}{c}\text { Bounded on } L_{p, \mu} \\
\text { Continuous on } \\
D_{p, \mu}\end{array}$ & $\begin{array}{c}a \in \mathbb{C} \\
b>0, \mu>0 \\
p \in(1, \infty)\end{array}$ \\
\hline \multirow[t]{2}{*}{$K_{b}^{a}$} & $e^{\mu t} W[\zeta,-\mu] e^{-\mu t}$ & $\underline{2(b / 4 s)^{a / 2} K_{a}\left(b^{1 / 2} s^{1 / 2}\right)}$ & Bounded on $L_{p, \mu}$ & $a \in \mathbb{C}$ \\
\hline & & $\begin{array}{c}\Gamma(a) \\
\operatorname{Re} s>0\end{array}$ & $\begin{array}{c}\text { Continuous on } \\
D_{p, \mu} .\end{array}$ & $\begin{array}{c}b>0, \mu<0 \\
p \in(1, \infty)\end{array}$ \\
\hline
\end{tabular}

\section{Remark 4.6.}

(a) Of particular interest in the sequel are the operators $I_{x^{2}}^{1 / 2}$ and $K_{x^{2}}^{1 / 2}$. By replacing $a$ and $b$ by $\frac{1}{2}$ and $x^{2}$ respectively in the appropriate entry of the above table, we find that the associated multiplier function is

$$
\begin{aligned}
\zeta(s) & =2\left(x^{2} / 4 s\right)^{1 / 4} K_{1 / 2}\left(|x| s^{1 / 2}\right) / \Gamma\left(\frac{1}{2}\right) \quad(\operatorname{Re} s>0) \\
& =s^{-1 / 2} e^{-|x| \sqrt{s}} .
\end{aligned}
$$

(b) For $\mu>0$, the operator $e^{\mu t} K^{a} e^{-\mu t}$ is the adjoint of $e^{-\mu t} I^{a} e^{\mu t}$ while $e^{\mu t} K_{b}^{a} e^{-\mu t}$ is the adjoint of $e^{-\mu t} I_{b}^{a} e^{\mu t}$. In each case, the operator and its adjoint can be written in 
multiplier from as $W\left[\zeta^{*}, \mu\right]$ and $W[\zeta, \mu]$ respectively (with the multiplier function chosen appropriately). This is not surprising since, for suitable $\psi$,

$$
F \zeta_{\sigma} F^{-1} \psi=F^{-1} \zeta_{\sigma}^{*} F \psi
$$

so that, in general, $W[\zeta, \mu]$ is the adjoint of $M\left[\zeta^{*}, \mu\right]$.

Theorem 4.7. Let $\phi \in D_{p, \mu}$, where $1<p<\infty$.

(a) If $\mu>0$ then $I_{b}^{1 / 2} \phi \rightarrow I^{1 / 2} \phi$ in $D_{p, \mu}$ as $b \rightarrow 0+$.

(b) If $\mu<0$ then $K_{b}^{1 / 2} \phi \rightarrow K^{1 / 2} \phi$ in $D_{p, \mu}$ as $b \rightarrow 0+$.

Proof. We prove (a) only, the proof of (b) being similar. If $\mu>0$ and $\phi \in D_{p, \mu}$ then

$$
\begin{aligned}
v_{k}^{p, \mu}\left(I_{b}^{1 / 2} \phi-I^{1 / 2} \phi\right) & =\left\|D^{k}\left(e^{-\mu t}\left[I_{b}^{1 / 2}-I^{1 / 2}\right] e^{\mu t} \psi\right)\right\|_{p} \quad\left(\psi \in D_{L, p}, \psi=e^{-\mu t} \phi\right) \\
& \leqq\left\|I_{b}^{1 / 2}-I^{1 / 2}\right\|\left\|D^{k} \psi\right\|_{p} \quad(\text { from }(3.5)) \\
& \rightarrow 0 \text { as } b \rightarrow 0+\quad \text { (from Theorem 4.1(c)) }
\end{aligned}
$$

Theorem 4.8. Let $\phi \in D_{p, \mu}$ with $1<p<\infty$.

(a) If $\mu>0$ then $I_{x^{2}}^{1 / 2} \phi$ is a twice strongly-differentiable $D_{p, \mu}$-valued function of $x$ for $x \neq 0$.

(b) If $\mu<0$ then $K_{x^{2}}^{1 / 2} \phi$ is a twice strongly-differentiable $D_{p, \mu}$-valued function of $x$ for $x \neq 0$.

Proof. (a) Let $T_{x}$ be the operator defined on suitable functions $\phi$ by

$$
\left(T_{x} \phi\right)(t)=-(x / 2 \sqrt{ } \pi) \int_{-\infty}^{t}(t-\tau)^{-3 / 2} \exp \left(-x^{2} / 4(t-\tau)\right) \phi(\tau) d \tau
$$

Since $T_{x}=x I_{x^{2}}^{-1 / 2}$, we can deduce that $T_{x}: D_{p, \mu} \rightarrow D_{p, \mu}$ is continuous and

$$
D^{k}\left(e^{-\mu t} T_{x} e^{\mu t} \psi\right)=e^{-\mu t} T_{x} e^{\mu t} D^{k} \psi
$$

for each $\psi \in D_{L^{p}}$ and $k=0,1, \ldots$. Consequently,

$$
\begin{aligned}
v_{k}^{p, \mu}\left(\left(h^{-1}\left[I_{(x+h)^{2}}^{1 / 2}-I_{x^{2}}^{1 / 2}\right]-T_{x}\right) \phi\right), \quad \phi \in D_{p, \mu} \\
\quad=\left\|e^{-\mu t}\left(h^{-1}\left[I_{(x+h)^{2}}^{1 / 2}-I_{x^{2}}^{1 / 2}\right]-T_{x}\right) e^{\mu t} D^{k} \psi\right\|_{p}, \quad\left(\psi=e^{-\mu t} \phi\right), \\
\leqq M(x, h, \mu) v_{k}^{p, \mu}(\phi) \quad \text { (using Young's inequality) }
\end{aligned}
$$

where $M(x, h, \mu)$ is the constant given by

$$
M(x, h, \mu)=\pi^{-1 / 2} \int_{0}^{\infty} w^{-1 / 2} e^{-\mu w}\left|h^{-1}\left(e^{-(x+h)^{2} / 4 w}-e^{-x^{2} / 4 w}\right)+(2 w)^{-1} x e^{-x^{2} / 4 w}\right| d w .
$$


Suppose without loss of generality that $h \in(-\varepsilon, \varepsilon)$ where $\varepsilon>0$ is chosen small enough to ensure that $[x-\varepsilon, x+\varepsilon] \subset \mathbb{R}-\{0\}$. By the second mean value theorem, there exists $\xi(=\xi(w, h))$ in $(x-\varepsilon, x+\varepsilon)$ such that

$$
e^{-(x+h)^{2} / 4 w}=e^{-x^{2} / 4 w}-(2 w)^{-1} h x e^{-x^{2} / 4 w}+2^{-1} h^{2} e^{-\xi^{2} / 4 w}\left[\left(\xi^{2} / 4 w^{2}\right)-\left(\frac{1}{2} w\right)\right] .
$$

Hence,

$$
\begin{aligned}
\mid h^{-1}\left(e^{-(x+h)^{2} / 4 w}-e^{-x^{2} / 4 w}\right) & +(2 w)^{-1} x e^{-x^{2} / 4 w} \mid \\
& \leqq 2^{-1}|h| e^{-\xi^{2} / 4 w}\left|\left(\xi^{2} / 4 w^{2}\right)-\left(\frac{1}{2} w\right)\right| \\
& \leqq 2^{-1}|h| e^{-\eta / 4 w}\left(e^{\left[\eta-(x-\varepsilon)^{2}\right] / 4 w}\left[((x+\varepsilon) / 2 w)^{2}+\left(\frac{1}{2} w\right)\right]\right)
\end{aligned}
$$

if $x-\varepsilon>0$, where $\eta>0$ is chosen so that $\eta-(x-\varepsilon)^{2}<0$. Similarly, if $x+\varepsilon<0$ then the expression in (4.8) can be shown to be bounded above by

$$
2^{-1}|h| e^{-\eta / 4 w}\left(e^{\left[\eta-(x+\varepsilon)^{2}\right] / 4 w}\left[((x-\varepsilon) / 2 w)^{2}+\left(\frac{1}{2} w\right)\right]\right)
$$

where this time $\eta$ is chosen so that $\eta-(x+\varepsilon)^{2}<0$. In either case, we can deduce that a constant $C$ exists such that

$$
\begin{aligned}
M(x, h, \mu) & \leqq C|h| \int_{0}^{\infty} w^{-1 / 2} e^{-\mu w} e^{-\eta / 4 w} d w \\
& =C|h| \sqrt{\pi} \mu^{-1 / 2} e^{-\eta^{1 / 2} \mu^{1 / 2}}
\end{aligned}
$$

and this converges to 0 as $h \rightarrow 0$. As a result, $I_{x^{2}}^{1 / 2}$ is strongly-differentiable for $x \neq 0$ with $d / d x\left(I_{x^{2}}^{1 / 2}\right)=T_{x}$ as operators on $D_{p, \mu}$. In a similar manner, we can verify that $d^{2} / d x^{2}\left(I_{x^{2}}^{1 / 2}\right)=$ $T_{x}^{\prime}(x \neq 0)$ as operators on $D_{p, \mu}$ where, for $\phi \in D_{p, \mu}$,

$$
\left(T_{x}^{\prime} \phi\right)(t)=\pi^{-1 / 2} \int_{-\infty}^{t}\left[(t-\tau)^{-5 / 2}\left(x^{2} / 4\right)-2^{-1}(t-\tau)^{-3 / 2}\right] \exp \left(-x^{2} / 4(t-\tau)\right) \phi(\tau) d \tau
$$

(b) This can be proved in the same manner as (a).

Corollary 4.9. Let $1<p<\infty, \phi \in D_{p, \mu}$ and $t$ be a fixed point in $\mathbb{R}$.

(a) If $\mu>0$ then $\left(I_{x^{2}}^{1 / 2} \phi\right)(t)$ is twice continuously differentiable with respect to $x$ on $\mathbb{R}-\{0\}$.

(b) If $\mu<0$ then $\left(K_{x^{2}}^{1 / 2} \phi\right)(t)$ is twice continuously differentiable with respect to $x$ on $\mathbb{R}-\{0\}$.

Proof. If we note first that the singular distribution $\delta_{t}$ defined by $\left(\delta_{t}, \phi\right)=\phi(t)$ belongs to $D_{p, \mu}^{\prime}$ then these results follow from the fact that strong convergence implies weak convergence in a Fréchet space. 


\section{Multiplier operators on the spaces $D_{L p}^{\prime}$}

The operators $W[\zeta, \sigma]$ and $W\left[\zeta^{*}, \sigma\right]$ defined in Section 3 can be extended in a natural manner to the generalised function spaces $D_{L p}^{\prime}$. The method adopted to carry out this extension process is the adjoint operator approach described in [5]. To see what this entails, let $\psi$ belong to the Banach space $L^{q}$ and let $\alpha(\zeta)<\operatorname{Re} s<\beta(\zeta)$ so that, from Lemma 2.4 and Theorem 3.3, $\psi$ and $W[\zeta, \sigma] \psi$ generate regular generalised functions $\psi$ and $\widetilde{W[\zeta, \sigma] \psi}$ in $D_{L \text { p }}^{\prime}$. If we denote the extended version of $W[\zeta, \sigma]$ by $\tilde{W}[\zeta, \sigma]$, then we require

$$
\tilde{W}[\zeta, \sigma] \tilde{\psi}=\overparen{W[\zeta, \sigma] \psi}
$$

as generalised functions in $D_{L^{p}}^{\prime}$. Suppose temporarily that $\psi, \phi \in S$. Then

$$
\begin{aligned}
(\tilde{W}[\zeta, \sigma] \tilde{\psi}, \phi) & =\overparen{(W(\zeta, \sigma) \psi, \phi)} \\
& =\int_{-\infty}^{\infty}\left(F^{-1} \zeta_{\sigma} F \psi\right)(t) \phi(t) d t \\
& =\int_{-\infty}^{\infty} \psi(t)\left(F \zeta_{\sigma} F^{-1} \phi\right)(t) d t \quad \text { (by Parseval's equation [10, p. 179]) } \\
& =\int_{-\infty}^{\infty} \psi(t)\left(F^{-1} \zeta_{\sigma}^{*} F \phi\right)(t) d t \quad(\text { by }(4.7)) \\
& =\left(\tilde{\psi}, W\left[\zeta^{*}, \sigma\right] \phi\right) .
\end{aligned}
$$

Similarly, under the same restrictions on $\phi$ and $\psi$, we can show that

$$
\left(\tilde{W}\left[\zeta^{*}, \sigma\right] \tau, \phi\right)=\left(\widetilde{W\left[\zeta^{*}, \sigma\right] \psi}, \phi\right)=(\tilde{\psi}, W[\zeta, \sigma] \phi) .
$$

Motivated by this, we make the following definition.

Definition 5.1. If $\zeta \in \mathscr{A}$ and $\zeta^{*}$ is defined by (3.2) then, for any $\sigma \in(\alpha(\zeta), \beta(\zeta))$ and $p \in(1, \infty)$, the operators $W[\zeta, \sigma]$ and $W\left[\zeta^{*}, \sigma\right]$ are extended to $D_{L p}^{\prime}$ by the formulae

$$
\begin{aligned}
& (\tilde{W}[\zeta, \sigma] f, \phi)=\left(f, W\left[\zeta^{*}, \sigma\right] \phi\right), \quad f \in D_{L^{p}}^{\prime}, \phi \in D_{L^{\rho}} \\
& \left(\tilde{W}\left[\zeta^{*}, \sigma\right] f, \phi\right)=(f, W[\zeta, \sigma] \phi), \quad f \in D_{L p}^{\prime}, \phi \in D_{L p} .
\end{aligned}
$$

Using standard properties of adjoint operators (see [11]), we can immediately prove the following.

Theorem 5.2. Let $\zeta \in \mathscr{A}$ and let $W[\zeta, \sigma]$ and $W\left[\zeta^{*}, \sigma\right]$ be defined on $D_{L p}^{\prime}$ by (5.1) and (5.2) respectively. Then, for any $\sigma \in(\alpha(\zeta), \beta(\zeta))$ and $p \in(1, \infty), \tilde{W}[\zeta, \sigma]$ and $\tilde{W}\left[\zeta^{*}, \sigma\right]$ are continuous linear mappings of $D_{L p}^{\prime}$ into $D_{L p}^{\prime}$. 
Proof. This follows from the corresponding properties of $W\left[\zeta^{*}, \sigma\right]$ and $W[\zeta, \sigma]$ on $D_{L p}$.

\section{Fractional integrals on $D_{p, \mu}^{\prime}$}

In Section 4 it was established that each of the operators $I^{a}, D^{a}$ and $I_{b}^{a}$, on $D_{p, \mu}$, could be expressed as a product of the form $e^{\mu t} W\left[\zeta^{*}, \mu\right] e^{-\mu t}$ (see Table 4.5). The discussion given in the previous section indicates that the natural extensions of these operators to the spaces $D_{p, \mu}^{\prime}$ take the form $\widetilde{e^{-\mu t}} W\left[\zeta^{*},-\mu\right] \widetilde{e^{\mu t}}$, where $\widetilde{e^{\mu t}}$ and $\widetilde{e^{-\mu t}}$ are defined by (2.1). From (5.1) and (5.2), we can deduce that, for $f \in D_{p, \mu}^{\prime}, \phi \in D_{p, \mu}$ and the appropriate choice of multiplier function $\zeta$,

$$
\begin{aligned}
& \left(\tilde{I}^{a} f, \phi\right)=\left(f, e^{\mu t} W[\zeta,-\mu] e^{-\mu t} \phi\right)=\left(f, K^{a} \phi\right) \\
& \left(\tilde{D}^{a} f, \phi\right)=\left(f, e^{\mu t} W[\zeta,-\mu] e^{-\mu t} \phi\right)=\left(f, E^{a} \phi\right)
\end{aligned}
$$

and

$$
\left(\tilde{I}_{b}^{a} f, \phi\right)=\left(f, e^{\mu t} W[\zeta,-\mu] e^{-\mu t} \phi\right)=\left(f, K_{b}^{a} \phi\right)
$$

Note that formula (5.3) is simply a statement of the rule for fractional integration by parts and is the formula usually used for extending the classical operator $I^{a}$ to spaces of distributions. Formula (5.7) is also expected since the operators $I_{b}^{a}$ and $K_{b}^{a}$ are formal adjoints.

Proceeding similarly, we also arrive at the following formulae:

$$
\begin{aligned}
& \left(\tilde{K}^{a} f, \phi\right)=\left(f, I^{a} \phi\right), \\
& \left(\tilde{E}^{a} f, \phi\right)=\left(f, D^{a} \phi\right), \\
& \left(\tilde{K}_{b}^{a} f, \phi\right)=\left(f, I_{b}^{a} \phi\right)
\end{aligned}
$$

where, in each case, $f \in D_{p, \mu}^{\prime}$ and $\phi \in D_{p, \mu}$.

\section{Thorem 6.1.}

(a) If $\mu<0, \operatorname{Re} a>0$ and $p \in(1, \infty)$ then $\tilde{I}^{a}$ is a homeomorphism of $D_{p, \mu}^{\prime}$ onto $D_{p, \mu}^{\prime}$ with $\left(\tilde{I}^{a}\right)^{-1}=\tilde{D}^{a}$.

(b) If $\mu<0, a \in \mathbb{C}, b>0$ and $p \in(1, \infty)$ then $\tilde{I}_{b}^{a}$ is a continuous linear mapping of $D_{p, \mu}^{\prime}$ into $D_{p, \mu}^{\prime}$.

Proof. These facts follow immediately from the corresponding properties of $K^{a}, E^{a}$ and $K_{b}^{a}$ on $D_{p, \mu}$.

Similar results can also be established for $\widetilde{K}^{a}, \tilde{E}^{a}$ and $\widetilde{K}_{b}^{a}$. 
Theorem 6.2. Let $f \in D_{p, \mu}^{\prime}$ where $1<p<\infty$.

(a) If $\mu<0$ then $\widetilde{I}_{b}^{1 / 2} f \rightarrow \tilde{I}^{1 / 2} f$ in $D_{p, \mu}^{\prime}$ as $b \rightarrow 0+$.

(b) If $\mu>0$ then $\tilde{K}_{b}^{1 / 2} f \rightarrow \tilde{K}^{1 / 2} f$ in $D_{p, \mu}^{\prime}$ as $b \rightarrow 0+$.

Proof. We prove only (a), the proof of (b) being similar. Let $\phi$ be any test function in $D_{p, \mu}$. Then

$$
\begin{aligned}
\left(\left(\tilde{I}_{b}^{1 / 2}-\tilde{I}^{1 / 2}\right) f, \phi\right) & =\left(f,\left(K_{b}^{1 / 2}-K^{1 / 2}\right) \phi\right) \rightarrow\left(f,\left(K^{1 / 2}-K^{1 / 2}\right) \phi\right) \\
& =0 \text { as } b \rightarrow 0+\quad(\text { from Theorem } 4.7(b))
\end{aligned}
$$

and this proves the assertion.

Theorem 6.3. Let $f \in D_{p, \mu}^{\prime}$, where $1<p<\infty$.

(a) If $\mu<0$ then $\widetilde{I}_{x^{2}}^{1 / 2} f$ is a twice weak ${ }^{*}$-differentiable $D_{p, \mu^{\prime}}^{\prime}$-valued function of $x$ for $x \neq 0$.

(b) If $\mu>0$ then $\tilde{K}_{x^{2}}^{1 / 2} f$ is a twice weak-*differentiable $D_{p, \mu}^{\prime}$-valued function of $x$ for $x \neq 0$.

Proof. This can be proved in the same manner as the previous theorem by using the results given in Theorem 4.8 .

\section{Application to the heat equation}

In this final section, the preceding theory is applied to find distributional solutions of a simple class of boundary value problems involving the one-dimensional heat operator $\partial / \partial t-\partial^{2} / \partial x^{2}$. The classical formulation of a typical problem is as follows. Given $f(=f(t))$, find $u(=u(x, t))$ in $C^{2}(\Omega) \cap C(\Omega), \Omega=\{(x, t): x>0, t>0\}$, such that

$$
\begin{gathered}
\frac{\partial u}{\partial t}=\frac{\partial^{2} u}{\partial x^{2}} \quad \text { in } \Omega \\
u(0, t)=f(t), \quad t>0 \\
u(x, 0)=0, \quad x>0 .
\end{gathered}
$$

Under suitable restrictions on the function $f$, it is possible to show $[1, \mathrm{p} .240]$ that a solution of this problem is given by

$$
u(x, t)=(2 \sqrt{\pi})^{-1} \int_{0}^{t}(t-\tau)^{-1 / 2} \exp \left(-x^{2} / 4(t-\tau)\right) g(\tau) d \tau
$$

where the function $g$ satisfies the Abel integral equation

$$
(2 \sqrt{\pi})^{-1} \int_{0}^{t}(t-\tau)^{-1 / 2} g(\tau) d \tau=f(t), \quad t>0
$$


Alternatively, by applying the Laplace transform, a solution can be found in the form

$$
u(x, t)=x(2 \sqrt{\pi})^{-1} \int_{0}^{t}(t-\tau)^{-3 / 2} \exp \left(-x^{2} / 4(t-\tau)\right) f(\tau) d \tau
$$

(see [1, p. 243]).

Our objective is to demonstrate that similar results hold for the same problem posed within a distributional framework where fewer restrictions are necessary on the function $f$. The distributional version of (7.1)-(7.3) which we consider is the following. Given $f \in D_{p, \mu}^{\prime}$, find $u(x) \in D_{p, \mu}^{\prime}(x \geqq 0)$ such that

$$
\begin{gathered}
\frac{\tilde{\partial}}{\partial t} u(x)=\frac{\partial^{2}}{\partial x^{2}} u(x) \quad \text { in } \Omega \\
\lim _{x \rightarrow 0+} u(x)=f, \text { where } \operatorname{supp} f \subseteq[0, \infty) \\
\operatorname{supp} u(x) \subseteq[0, \infty) \text { for each } x>0 .
\end{gathered}
$$

The solution $u(x)$ we seek is regarded as a $D_{p, \mu}^{\prime}$-valued function of $x$ for $x \geqq 0$. Consequently, in (7.6), differentiation with respect to $x$ is interpreted as differentiation in the weak* sense of a $D_{p, \mu}^{\prime}$-valued function, whereas $\tilde{\partial} / \partial t$ denotes the distributional differential operator defined by

$$
\left(\frac{\partial}{\partial t} u(x), \phi\right)=\left(u(x),-\frac{d \phi}{d t}\right), \quad \phi \in D_{p, \mu} .
$$

In (7.7), convergence is with respect to the weak*-topology while in (7.8), support is interpreted in the distributional sense.

Lemma 7.1. If $\mu<0$ and $p \in(1, \infty)$ then a solution of $(7.6)$ and (7.7) is given by

$$
u(x)=\tilde{I}_{x^{2}}^{1 / 2}\left(\tilde{D}^{1 / 2} f\right)
$$

Proof. From Theorems 6.1(a) and 6.2(a),

$$
\tilde{I}_{x^{2}}^{1 / 2}\left(\tilde{D}^{1 / 2} f\right) \rightarrow \tilde{I}^{1 / 2}\left(\tilde{D}^{1 / 2} f\right)=f \text { as } x \rightarrow 0+
$$

and therefore $u(x)$, given by (7.9), satisfies the boundary condition (7.7). Moreover, from Theorem $6.3(\mathrm{a}), \widetilde{I}_{x^{2}}^{1 / 2}\left(\tilde{D}^{1 / 2} f\right)$ is twice weak ${ }^{*}$ differentiable with respect to $x$ for $x>0$. To complete the proof, it remains to show that

$$
\frac{\partial^{2}}{\partial x^{2}}\left[\tilde{I}_{x^{2}}^{1 / 2}\left(\tilde{D}^{1 / 2} f\right)\right]=\frac{\partial}{\partial t}\left[\tilde{I}_{x^{2}}^{1 / 2}\left(\tilde{D}^{1 / 2} f\right)\right]
$$


Let $\phi \in D_{p, \mu}$ and let $g=\widetilde{D}^{1 / 2} f$. Then $g \in D_{p, \mu}^{\prime}$ and, by definition,

$$
\left(\frac{\partial}{\partial t}\left(\tilde{I}_{x^{2}}^{1 / 2} g\right), \phi\right)=-\left(g, K_{x^{2}}^{1 / 2}(d \phi / d t)\right) .
$$

Now

$$
\begin{aligned}
{\left[K_{x^{2}}^{1 / 2}(d \phi / d t)\right](t) } & =\pi^{-1 / 2} \int_{t}^{\infty}(\tau-t)^{-1 / 2} \exp \left(-x^{2} / 4(\tau-t)\right)(d \phi / d \tau)(\tau) d \tau \\
& =-\pi^{-1 / 2} \int_{t}^{\infty}\left[(\tau-t)^{-5 / 2}\left(x^{2} / 4\right)-(\tau-t)^{-3 / 2} 2^{-1}\right] \exp \left(-x^{2} / 4(\tau-t)\right) \phi(\tau) d \tau
\end{aligned}
$$

on integrating by parts. Similarly,

$$
\left(\frac{\partial^{2}}{\partial \mathrm{x}^{2}}\left(\tilde{I}_{x^{2}}^{1 / 2} g\right), \phi\right)=\left(g, \frac{\partial^{2}}{\partial x^{2}}\left(K_{x^{2}}^{1 / 2} \phi\right)\right)
$$

and, on differentiating under the integral sign twice with respect to $x$, we find that $-\partial^{2}\left(K_{x^{2}}^{1 / 2} \phi\right) / \partial x^{2}$ is given by (7.11). This holds for any $x>0$ and hence the theorem follows immediately.

Lemma 7.2. If $\mu<0$ and $p \in(1, \infty)$ then the solution $u(x)$ of $(7.6)$ and (7.7), given by (7.9), has the equivalent representation

$$
u(x)=-x \tilde{I}_{x^{2}}^{-1 / 2} f
$$

Proof. For $\phi \in D_{p, \mu}^{\prime}$,

$$
\left(-x \widetilde{I}_{x^{2}}^{-1 / 2} f, \phi\right)=\left(f,-x K_{x^{2}}^{-1 / 2} \phi\right)
$$

and

$$
\left(\widetilde{I}_{x^{2}}^{1 / 2}\left(\widetilde{D}^{1 / 2} f\right), \phi\right)=\left(f, E^{1 / 2}\left(K_{x^{2}}^{1 / 2} \phi\right)\right)
$$

Therefore the result will follow if we can show that

$$
E^{1 / 2}\left(K_{x^{2}}^{1 / 2} \phi\right)=-x K_{x^{2}}^{-1 / 2} \phi, \quad \phi \in D_{p, \mu}
$$

This identity is best established by considering the corresponding multiplier functions. On the left-hand side, $E^{1 / 2} K_{x^{2}}^{1 / 2}$ corresponds to $s^{1 / 2} s^{-1 / 2} \exp \left(-x s^{1 / 2}\right)$ while on the righthand side the corresponding multiplier function is $(x / 2 \sqrt{ } \pi)\left(2 \sqrt{ } \pi x^{-1} \exp \left(-x s^{1 / 2}\right)\right.$ ) (see Table 4.5, Remark 4.6(a) and [2, p. 146, (28)]). Hence, in each case the associated multiplier is $\exp \left(-x s^{1 / 2}\right)$ so that (7.13) is certainly true on the dense subset $S$ of $D_{p, \mu}$. The result for any $\phi \in D_{p, \mu}$ follows from the continuity of the operators. 
Lemma 7.3. If $\mu<0, p \in(1, \infty)$ and supp $f \subseteq[0, \infty)$ then supp $u(x) \subseteq[0, \infty)$ for each $x>0$, where $u(x)$ is given by either (7.9) or (7.12).

Proof. Let $\phi \in D_{p, \mu}$ be such that $\operatorname{supp} \phi \subset(-\infty, 0)$. Then

$$
\begin{aligned}
(u(x), \phi) & =-x\left(\tilde{I}_{x^{2}}^{-1 / 2} f, \phi\right) \\
& =-x\left(f, K_{x^{2}}^{-1 / 2} \phi\right) \\
& =0
\end{aligned}
$$

where the last line follows since supp $f \subseteq[0, \infty)$ and $\operatorname{supp} K_{x^{2}}^{-1 / 2} \phi \subset(-\infty, 0)$.

As a consequence of Lemmas 7.1-7.3, it is seen that a solution of (7.6)-(7.8) always exists in $D_{p, \mu}^{\prime}$ whenever $1<p<\infty$ and $\mu<0$. This solution can be expressed as either (7.9) or (7.12).

Finally, it should be noted that, with slight modifications, the above analysis will provide distributional solutions of problems involving different boundary conditions (e.g. $\partial u / \partial x+\lambda u=f(t)$ at $x=0$ ) and can also be used to deal with spherically symmetric problems involving the three-dimensional heat operator. It is the author's intention to discuss these additional problems in a future paper.

\section{REFERENCES}

1. E. T. Copson, Partial differential equations (Cambridge University Press, 1975).

2. A. ERdélyi et al., Tables of integral transforms (Vol. 1) (McGraw-Hill, New York, 1954).

3. W. LAmb, A distributional theory of fractional calculus, Proc. Royal Soc. Edinburgh 99A (1985), 347-357.

4. N. N. LeBedev, Special functions and their applications (Dover, New York, 1972).

5. A. C. MCBride, Fractional calculus and integral transforms of generalized functions (Research Notes in Mathematics 31, Pitman, London, 1979).

6. G. O. OKIxIOLU, Aspects of the theory of bounded integral operators in $L^{p}$-spaces (Academic Press, London, 1971).

7. P. G. Rooney, On the ranges of certain fractional integrals, Canad. J. Math. 24 (1972), 1198-1216.

8. P. G. Rooney, A technique for studying the boundedness and extendability of certain types of operators, Canad. J. Math. 25 (1973), 1090-1102.

9. L. Schwartz, Théorie des distributions (Hermann, Paris, 1966). 1965).

10. A. H. Zemanian, Distribution theory and transform analysis (McGraw-Hill, New York,

11. A. H. Zemanian, Generalized integral transformations (Interscience, New York, 1968).

Department of Mathematics

UNIVERSITY OF STRATHCLYDE

GlasGow 